\title{
O PERFIL DO ATENDIDO E DOS PROFISSIONAIS QUE COMPÕE O TRATAMENTO DAS DROGAS EM COMUNIDADES TERAPÊUTICAS
}

DOI: $10.22289 / 2446-922 X . V 2 N 2 A 7$

\author{
Mayza Caetano de Souza \\ Gilmar Antoniassi Júnior ${ }^{1}$
}

\section{RESUMO}

O estudo objetivou identificar o perfil dos usuários em tratamento e a condição de envolvimento de álcool e outras drogas bem como a equipe de profissional disponibilizada em comunidades terapêuticas (CT). Trata-se de um estudo qualitativo, descritivo, de natureza exploratória realizado em três instituições. Utilizou-se um Questionário elaborado para identificação do perfil do atendido e dos profissionais das comunidades terapêuticas. Os resultados apontam que não existe um protocolo especifico de ações e que, cada CT, tendese a agir com sua própria filosofia institucional. A principal droga apontada como eliciadora da internação dos residentes pelas CT corresponde à dependência do uso de Crack e Álcool. Dos profissionais envolvidos na CT, 90,9\% buscaram se profissionalizar através de leituras e capacitações. Conclui-se que há necessidade de os profissionais das CT's estudadas ampliarem o conhecimento sobre propostas melhor delineadas de tratamento para os dependentes de álcool e outras drogas.

Palavras-Chave: Comunidade Terapêuticas, Drogas, Tratamento.

\section{ABSTRACT}

The objective of this study is to identify the treating user profile and his involving with alcohol and other drug, as well as the professional team available in therapeutic communities. It was a qualitative study of the descriptive type of exploratory nature, carried out in three institutions. A questionnaire was elaborated to identify the profile of the attendee and the professionals of the therapeutic communities (TC). The results indicate that there is no specific actionsprotocol, and that each TC tends to act with its own institutional philosophy. The main drug indicated as hospitalization elicitors of the residents by the TC corresponds to the dependence of the use of crack and alcohol. From professionals involved in the TC, 90.9\% pursued to become professional, through readings and training. It is concluded that there is a need of the referred TC professionals to increase knowledge about better-designed treatment proposals for alcohol and other drug dependents.

Keywords: Community Therapeutics, Drugs, Treatment.

\footnotetext{
${ }^{1}$ Endereço eletrônico de contato: jrantoniassi@hotmail.com
}

Recebido em 16/12/2016. Aprovado pelo Conselho Editorial e aceito para publicação em 23/12/2016. 


\section{INTRODUÇÃO}

Em decorrência dos dias atuais, em que o uso de drogas vem a cada dia aumentando, não existe qualquer contexto no qual o uso não ocorra. $O$ álcool tem sido a droga mais consumida pela população. Por diversas finalidades a prática do uso de drogas é universal e milenar. Em algumas sociedades, o uso tem relação com a divindade e é usada como facilitador da comunicação com espíritos, por exemplo. Porém, a partir dos anos 60, o uso das drogas tornou-se uma preocupação para a sociedade. ${ }^{(1)}$

A influência do capitalismo na representatividade do indivíduo que vive em sociedade tem se mostrado o dogma do que se tem. As pessoas parecem viver para ter, o que aponta para a necessidade exacerbada do consumismo imediato visando satisfazer suas necessidades individuais. É nessa busca incessante do imediatismo na resolutividade de problemas e a partir da busca inconsciente da auto aceitação, da necessidade de pertencer a um estigma de padrão social a fim de legitimar-se a um grupo que as pessoas acabam por fazer escolhas "inadequadas" perante a extensa oferta que o capitalismo nos oferece no que diz respeito ao uso das drogas. ${ }^{(2)}$

O uso exagerado das drogas e a forma de tratamento apontam para os novos desafios que a área da saúde vem enfrentando em torno da temática. Assim, o uso das drogas figura como o grande fenômeno contemporâneo que leva ao consumo excessivo podendo chegar à possível dependência. As proporções tomadas pelo uso desregrado do consumo das drogas são expressivas no Brasil e no mundo e a epidemiologia do crack faz com que a diversificação das ofertas de tratamento terapêutico aos dependentes se alastre de uma forma desordenada, apontando a necessidade de investimentos em políticas públicas. ${ }^{(3)}$

Ações de políticas precisam ser pensadas no âmbito em que sejam fortalecidas as redes de assistência, porém associadas às questões de à saúde, a fim de promover a saúde e prevenir o uso abusivo e a dependência da droga, possibilitando àquele individuo dependente a reinserção social e a reabilitação. ${ }^{(4)}$

O problema em torno das drogas é alarmante porque pelo menos $10 \%$ das populações dos centros urbanos consomem abusivamente drogas, independente de idade, grau de instrução, sexo ou posição social. Fato é que o álcool é a droga mais consumida entre a população geral, o uso nocivo e a dependência são dois tipos de problemas comuns e característicos daqueles que o utilizam. De acordo com o I Levantamento sobre os padrões de consumo de álcool na população brasileira com indivíduos de 18 anos ou mais, indicou-se que $3 \%$ têm problemas de uso nocivo, e $9 \%$ de dependência. Dados estes que são compatíveis com pesquisas anteriores que revelam que $12 \%$ da população brasileira tem

Rev. Psicol Saúde e Debate. Jan., 2017:2(2):112-123. 
algum problema relacionado ao álcool o que torna evidente a necessidade de políticas públicas de saúde com o custo social, pois é evidente o prejuízo social que a droga traz a sociedade. (5)

A partir dos problemas sociais devido ao uso exagerado das drogas e da necessidade de pensar de forma ampla o Brasil, por meio do decreto ํo. 7.179 de 20 de maio de 2010, instituiu o Plano Integrado de enfrentamento ao crack e outras drogas cujo objetivo é prevenir o uso, favorecer o tratamento e a reinserção social dos usuários buscando enfrentar o tráfico drogas. ${ }^{(6)}$

Em dezembro de 2011 foi lançado pelo Governo Federal o Programa Crack, é Possível Vencer, sob o tríplice eixo: Prevenção, Cuidado e Autoridade. Através destas medidas, por intermédio do Ministério da Justiça e devido à carência de informações, foi feito um levantamento e estudo para caracterizar quem são esses usuários, para que se tornem mais eficazes as diretrizes das políticas públicas tanto no que diz respeito à prevenção como ao tratamento a ser oferecido a estes dependentes. ${ }^{(7)}$

O uso do crack e de outras drogas hoje no Brasil tem se tornado o terror da população considerando-se que o perfil do usuário é de jovens com idade média de até 30 anos, do sexo masculino, solteiro e que cursou entre a $4^{\underline{a}}$ e $8^{\underline{a}}$ do ensino fundamental. Estudos realizados pela Secretaria Nacional Antidrogas apontam que 47,3\% dos usuários das capitais se encontra morando na rua e não tem residência fixa. Dado relevante é o uso simultâneo de outras drogas, sendo $80 \%$ o álcool e o tabaco são os mais associados. (5)

Vários são os motivos que podem levar ao uso de drogas, à vontade e a curiosidade em sentir o efeito preponderante ocasionado pelo uso, são fatores que tendem a motivar o indivíduo. Mediante a isso se abre a necessidade de repensar como está sendo tratada à questão de promoção e prevenção ao uso a partir da necessidade de refletir e evitar o primeiro contato a fim de resgatar os valores familiares e reforçando ações preventivas que vão desde a infância até a idade adulta. ${ }^{(8)}$

A droga pode ser considerada um dos recursos mais eficazes para o sujeito com o mal-estar inerente ao ser humano já que proporciona, além do prazer, um distanciamento do mundo externo, levando-o para uma realidade inversa à sua própria realidade, produzindo alívio de algo que está lhe incomodando e fazendo isso de forma prazerosa. (9) A grande problemática está na relação que o indivíduo tem com o consumo de álcool e de outras drogas, que tipo de relação é essa, no consumo de forma abusiva que provavelmente levará a dependência, trazendo prejuízos significativos tanto no seu contexto familiar, social, físico e psíquico. ${ }^{(10)}$

Rev. Psicol Saúde e Debate. Jan., 2017:2(2):112-123. 
A qualidade de vida das populações pode se fortalecer quando a ação da saúde pública se utiliza de serviços tecnológicos em saúde associados à vontade política. É preciso utilizar de todos os recursos disponíveis para atingir e sensibilizar a população sobre as consequências do uso do álcool e de outras drogas. ${ }^{(11)}$

A dependência é classificada como um transtorno psiquiátrico, onde é caracterizada por um processo lento e gradual. ${ }^{(12)}$ Tomando como base o DSM-IV (2002) diagnosticar a dependência consiste na presença de vários fatores baseados em sintomas cognitivos, comportamentais e fisiológicos. Definido como um conjunto de três ou mais dos sintomas, ocorre em qualquer momento, no mesmo período de 12 meses. ${ }^{(13)}$

É necessário que se reconheça o movimento biopsicossocial das dependências químicas, que é fruto de uma interação que está em uma constante dinâmica de três fatores distintos: o tipo de substância que é consumida e a consideração do indivíduo em seu meio social e familiar, ressalvando que um tipo de substancia pode ter efeito diferentes em diferentes usuários. Acredita-se que quanto mais possibilidades de apoio familiar, social e acesso a politicas publicas apresentadas ao sujeito enquanto fator protetivo, o risco de se criar um a dependência química diminui e também, possivelmente, o grau da dependência, mesmo que este mesmo sujeito já tenha consumido ou experimentado a droga. Dessa forma, as ações da rede pública de saúde devem ser fortalecidas para que haja uma oferta de tratamentos mais eficazes aos usuários sem desconsiderar as ações voltadas para a prevenção. ${ }^{(14)}$

As comunidades terapêuticas surgiram diante das lacunas assistenciais do SUS quando alguns setores da sociedade civil formados por abrigos e instituições filantrópicas começaram a oferecer apoio ao dependente e seus familiares utilizando como principal instrumento terapêutico a convivência entre os pares residentes. ${ }^{(15)}$

A discussão sobre o tratamento nas comunidades terapêuticas está em pauta pelos serviços público e privados quando se trata da ordem do tratamento de recuperação disponível na sociedade brasileira se tornando cada vez mais frequente nos meios de comunicação atuais. Em uma recente pesquisa feita pelo CFP (Conselho Federal de Psicologia) identificou-se que a maioria das Ct's analisadas, não dispõe de uma metodologia de tratamento que siga o modelo previsto pela ANVISA (Agência Nacional de Vigilância Sanitária) e pelo CONAD (Conselho Nacional Anti Drogas). ${ }^{(16)}$

O foco de tratamento da dependência em comunidades terapêuticas é de propor a transformação na subjetividade do sujeito, procurando resgatar seus valores através de uma intervenção direta nas características da personalidade do dependente, favorecendo sua reinserção social, evocando novos valores como a religiosidade, responsabilidade, 
solidariedade, honestidade e amor. As comunidades terapêuticas baseiam-se no modelo de tratamento residencial, fundamentadas na premissa de que quando o sujeito não consegue promover mudança em seus comportamentos quanto ao vício é necessário que se remova das situações que estimulam ao uso, ou seja, retirar do convívio facilitador ao uso e aumentar o distanciamento das drogas proporcionando o enfrentamento à doença. ${ }^{(10,17)}$

Contudo, se faz necessário repensar as posturas adotadas nestas comunidades, pois os serviços terapêuticos não podem ficar somente no afastamento, na eliminação da droga do organismo ou reversão das alterações neuropatológicas. Deve-se atentar para os aspectos psicológicos e socioculturais e para o fortalecimento do indivíduo com a proposta do tratamento, promovendo assim sua conscientização de que é tratamento para o seu bemestar, favorecendo a qualidade de vida e sua reinserção social. $(18,19)$

O presente trabalho tem por justificativa, frente às diversas tentativas de internação para tratamento de usuários de drogas, que são resultantes do fracasso na manutenção/ eu redução dos danos, chamar atenção no âmbito das estratégias que cada instituição dispõe, demonstrando que cada uma possui um modelo próprio e poucas são as instituições que usam dos aspectos biopsicossociais da concepção do indivíduo para fortalecer o enfrentamento e fortalecer a tentativa de superação das drogas no tratamento.

Neste sentido que o estudo se objetiva em identificar o perfil dos usuários em tratamento e a condição de envolvimento de álcool e outras drogas bem como a equipe profissional disponibilizada em comunidades terapêuticas.

\section{METODOLOGIA}

Trata-se de um estudo qualitativo do tipo descritivo de natureza exploratória realizado em três Comunidades Terapêuticas, de uma cidade da região do Alto Paranaíba do Estado de Minas Gerais. Os critérios de inclusão para pesquisa pautaram-se no fato de estarem devidamente cadastradas na Agência Nacional de Vigilância Sanitária (ANVISA) e por adesão mediante o contato prévio junto aos dirigentes responsáveis mediante apresentação da intenção de estudo. É válido ressaltar que, no munícipio, constam quatro comunidades cadastradas na ANVISA, porém, uma não aderiu à participação.

O estudo atendeu aos princípios éticos segundo Resolução do CNS 466/2013 para pesquisa com seres humanos. Para tanto, foi submetido através de documentação necessária 
para análise ética e acompanhamento do Comitê de Ética em Pesquisa (CEPE) da Universidade de Franca (Unifran), sob número 1235.256.

Para coleta de dados todos os participantes envolvidos foram convidados e receberão o Termo Consentimento de Livre Esclarecido (TCLE), onde constaram todas as informações e esclarecimentos necessários referentes ao estudo em questão. Foi utilizado Questionário elaborado para identificação do perfil do atendido e dos profissionais das comunidades terapêuticas e, para o procedimento de análise estatística dos dados obtidos no instrumento, foi utilizado o auxílio do programa Epilnfo ${ }^{\circledR}$ versão 3.5.2. (20)

\section{RESULTADOS E DISCUSSÃO}

A propósito disso, constatou-se que as exigências jurídicas para fundar uma CT devem fundamentar-se nas diretrizes expostas na Resolução - RDC № 29, de 30 de junho de 2011. (21)

Foram identificados no estudo total de $\mathrm{n}=116$ pacientes atendidos nas Comunidades Terapêuticas (CT's) denominadas CT-1, CT-2 e CT-3, sendo 100\% residentes nas CT's e $\mathrm{n}=64$ reincidentes, ou seja, que estão pela segunda ou terceira vez reincidindo na CT sendo as comunidades $100 \%$ masculinas.

A principal droga apontada como eliciadora da internação dos residentes pelas CT corresponde à dependência do uso de Crack e Álcool sendo o tempo médio de permanência nas CT para tratamento corresponde a 6 meses de internação para as três CT's.

Nesse sentido, comunga-se o estudo de Schnorr e Hess (2012) que investigou as perspectivas do usuário de crack sobre a sua reinserção social após o término de seu tratamento em comunidade terapêutica. Evidenciou-se que trabalhar com a dependência química requer atenção às expectativas da família que emergem como objetivos principais desse período de readaptação. ${ }^{(25)}$ A permanência tradicional na CT era de 12 a 18 meses, mas esse tempo de duração tem se modificado atualmente, pois, para as CT's afiliadas na FEBRACT, a permanência padrão pode variar entre 6 e 9 meses. (22)

Torna-se necessário que não seja negligenciada a necessidade de disponibilizar um profissional com qualquer curso de nível superior para ser o responsável técnico bem como um substituto para este profissional com a mesma qualificação. Cada residente deverá ter uma ficha individual para serem registradas informações referentes ao cotidiano, como atividades físicas e lúdicas, rotinas de estudo, atendimento às famílias etc. Também é preciso

Rev. Psicol Saúde e Debate. Jan., 2017:2(2):112-123. 
vetar o ingresso de pessoas cuja condição solicite serviços de saúde não oferecidos pela CT. Ainda é necessário especificar em suas normas o tempo máximo de permanência do residente na entidade, garantir respeito à pessoa e à família, de todas as etnias, religiões, ideologia, nacionalidade, orientação sexual, precedentes criminais ou condição financeira, garantir a permanência voluntária e atender os pré-requisitos de segurança sanitária. ${ }^{(21)}$

No que se refere aos profissionais, identificou-se $n=11$ envolvidos nas CT's, distribuídos em: CT-1 ( $n=3)$, CT-2 $(n=4)$ e CT-3 $(n=4)$, distribuídos na função de Médico; Psicólogo; Enfermeiro; Terapeuta e Coordenador. A tabela 1 possibilita verificar a comparação do profissional e sexo correspondente.

Tabela 1 - Distribuição dos profissionais por comunidade terapêutica

\begin{tabular}{lll}
\hline Atividade & $\begin{array}{l}\text { Masculino } \\
{[\mathrm{n}]}\end{array}$ & $\begin{array}{l}\text { Feminino } \\
{[\mathrm{n}]}\end{array}$ \\
\hline Psicologia $^{(\mathrm{n}=3)}$ & 1 & 2 \\
\hline Medicina $^{(\mathrm{n}=2)}$ & 2 & - \\
\hline Coordenação $^{(\mathrm{n}=2)}$ & 2 & - \\
\hline Terapeuta $^{(\mathrm{n}=2)}$ & 2 & 2 \\
\hline Enfermagem $^{(\mathrm{n}=2)}$ & - & \\
\hline
\end{tabular}

Em relação às políticas públicas norteadas para esta área, às comunidades terapêuticas e os conselhos municipais sobre drogas são providos de especificidades para o enfrentamento desta questão que necessitam ser articuladas e operacionalizadas em função de um atendimento adequado e interdisciplinar. A maior parte das CTs baseia-se em modelos fundamentalmente morais de tratamento da dependência de substâncias, essa filosofia unifica princípios de convivência comunitária e inserção social que dialogam com as atuais políticas públicas em Atenção Integral a Usuários de Álcool e outras Drogas. Assim, parece ser necessário não desprezar as possibilidades que essa modalidade de tratamento oferece, mas viabilizar meios de aprimorar suas intervenções. ${ }^{23,24)}$

Quanto ao envolvimento dos profissionais acerca do tratamento da dependência química, $\mathrm{n}=7$ dos profissionais envolvidos corresponde entre 1 a 6 anos de experiência e apenas $n=1$ profissional possui mais de 10 anos de experiência e $n=2$ profissionais a menos de 2 anos sendo que o meio de aprimoramento na área ocorre através de leituras e Rev. Psicol Saúde e Debate. Jan., 2017:2(2):112-123. 
capacitações para 90,9\% dos profissionais envolvidos e 9,1\% não buscam nenhum tipo de capacitação.

É valido ressaltar que, no estudo realizado por Schnorr e Hess, uma das preocupações evidentes dizia respeito à necessidade de resgatar e obter a aceitação da família do dependente, em auxiliar a conseguir novas amizades e afastar-se das antigas companhias de uso de drogas, no âmbito em que se sugeriu a importância de desenvolver novas técnicas para ajudar na fase de recuperação deste tipo de usuário. (25)

No entanto, os profissionais devem buscar trabalhar com o dependente químico da melhor forma possível por meio de ações empíricas cuja proposta seja criar um ambiente acolhedor e partidário a ele, que possa envolver sua família. Também é preciso aproveitar diferentes oportunidades para abordar o tema e a sua repercussão na vida dos envolvidos por meio das queixas e dificuldades que são componentes catalisadores de aproximação do dependente químico com a unidade e com os profissionais. A análise das falas conduz à reflexão da necessidade de uma qualificação da equipe por meio de medidas de uma educação continuada para atuar junto ao dependente químico. Entretanto, é urgente a necessidade de haver apoio de uma rede de Saúde Mental bem estruturada, hábil a oferecer respaldo às demandas inerentes a esse contexto de modo a elaborar propostas concretas para dinamizar essa área. (26)

O estudo evidencia que o envolvimento do profissional com a CT corresponde para $45,5 \%$ dos profissionais uma atuação exclusiva a CT e 54,5\% praticam atividades esporádicas e com horários limitados e previamente definidos. Neste contexto, $18 \%$ dos profissionais que estão envolvidos no tratamento revelam que a capacitação profissional periódica, a integração entre equipe e família do dependente e a discussão de caso na equipe e participação em congresso são fundamentais para a qualidade do tratamento ofertado na CT. $36 \%$ dos profissionais indicam que apenas uma destas possibilidades basta e para $46 \%$ é necessário pelo menos a prática de duas ou três delas para manter a qualidade.

Para que as atividades nas CT's possam promover mudanças é preciso entender o grupo social a ser trabalhado, isto é, compreender a dinâmica que os usuários produzem em seu ambiente, seus valores e símbolos. Compreender os residentes subjetivamente a fim de identificar as dificuldades e potencialidades para levar a pessoa ao viver sóbrio. As atividades devem permitir que os internos sejam agentes de sua transformação biopsicossocial. Devemse identificar os seus fatores motivadores, "[...] à experiência de consumo das substâncias psicoativas (se há relação com o sentido da vida, pertencimento a um grupo social, despreparo intelectual ou formação humana). [...]" (BARROS, 2012, p. 08). Para atuar dentro 
de qualquer CT's, as políticas públicas não podem ser padronizadas. Elas devem ser descentralizadas, de modo a respeitar as suas particularidades. ${ }^{(27)}$

Ao trabalhar com o dependente quando este se encontra em recuperação, valorizando o preparo para inserção social e cuidando para a elaboração do sujeito a recaídas, reforça-se que esse retorno ao convívio familiar e social, muitas vezes, não acontece com a tranquilidade necessária ao equilíbrio emocional do egresso ao iniciar essa nova etapa de sua vida. Isso, possivelmente, irá gerar uma expectativa de ambos os lados sendo importante destacar a necessidade de fortalecer, para o dependente em recuperação, a busca de novas relações sociais que, muitas vezes, são produzidas a partir de vínculos com as igrejas e seus (21,28).

Os motivos que levaram os profissionais estarem envolvidos no tratamento da dependência química em CT é configurado pela vontade de ajudar e auxiliar o outro no processo de reabilitação. Evidenciada através do relato:

COORD. 1 - ver a felicidade durante o tratamento é o principal motivo.

COORD. 2 - o próprio envolvimento com as drogas e a necessidade de auxiliar as pessoas a manter a sobriedade.

ENF. 1 - pelo fato de questões familiares e por gostar muito do tema.

ENF. 2 - flexibilidade de locais, abertura do mercado de trabalho.

MÉD. 1 - apenas como oportunidade de trabalho.

MÉD. 2 - por tratar-se de atividade ligada a minha área de atuação profissional.

PSIC. 1 - os motivos que me levaram a trabalhar com a dependência química, são por estar relacionado com a atenção psicossocial perante a demanda do usuário de álcool e outras drogas.

PSIC. 2 - além de ser uma área que pode proporcionar um bom retorno financeiro, existe também a questão humana envolvida, auxiliar uma pessoa que passa por problema através do uso das drogas é extremamente satisfatório.

PSIC. 3 - a afinidade com a área, o aprendizado proporcionado em campo, a ampliação de experiências com os usuários e a condição de capacitação contínua na modalidade de estudo de casos diferenciados e ao mesmo tempo comuns.

TERP. 1 - gosto do desafio sinto que sou capaz de contribuir de maneira positiva ao tratamento desses pacientes.

TERP. 2 - eu tenho um caso dentro de casa, hoje reabilitado, o que fez surgir o interesse de ajudar outros.

Rev. Psicol Saúde e Debate. Jan., 2017:2(2):112-123. 
Contudo, no processo de reinserção social do interno é fundamental o papel da família e/ou núcleo de pessoas com quem o mesmo tem vínculos afetivos. É essencial que o interno tenha uma referência afetiva, que possa garantir "para quem" e "para onde" voltar. O desligamento institucional é angustiante para os internos desprovidos dessa referência e, por isso, a CT deve intermediar as suas relações afetivas, do internamento, ao desligamento, até o regresso ao convívio familiar. Toda a equipe, sobretudo o assistente social e o psicólogo, deve atuar no sentido de fortalecer o convívio familiar lembrando-se ainda da importância da reinserção do egresso nos âmbitos educacional e profissional para que o mesmo possa se inserir na sociedade e praticar em plenitude o exercício da cidadania. ${ }^{(27)}$

\section{CONSIDERAÇÕES}

Foi possível constatar que não há um protocolo especifico de ações nas comunidades terapêuticas e que cada uma delas tende a agir com sua própria filosofia institucional, como os 12 passos do NA, prevenção de recaída, terapia racional emotiva, ações de ressocialização, acompanhamento médico, psicológico, serviços de contenção, trabalhos que envolvem a espiritualidade e o emocional do indivíduo. No entanto, observou-se que, para obter respostas mais eficazes, as propostas de intervenção nas comunidades terapêuticas devem ser fundamentadas em diferentes abordagens: médico-farmacológica, psicossocial, sociocultural e religiosa.

Conclui-se ser fundamental importância propor mais estudos referentes ao trabalho dos profissionais envolvidos em comunidades terapêuticas de modo a ampliar o conhecimento a respeito do que pode ser proposto para o tratamento dos dependentes de álcool e de outras drogas.

\section{REFERÊNCIAS}

1. Organización Mundial de La Salud. Conclusiones y recomendaciones de las Discusiones Técnicas sobre la Salud de los Jóvenes de la 42a Asamblea Mundial de la Salud, Ginebra; 1989 (p.3)

2. Silva JL. Terapia de rede para adictos: Programa de tratamento e prevenção para dependentes de drogas em comunidades terapêuticas. São Paulo, 2011. p.23.

Rev. Psicol Saúde e Debate. Jan., 2017:2(2):112-123. 
3. Brasil. Ministério da Saúde: Secretaria de Atenção à Saúde. SVS/CN-DST/AIDS. A Política do Ministério da Saúde para Atenção Integral a Usuários de Álcool e outras Drogas. 2ª . ed. ver. Ampl. Brasília, 2004.

4. Vieira E. Política econômica e política social. In: Os direitos e a política pública social. São Paulo: Cortez; 2004.

5. Brasil. I Levantamento Nacional sobre os padrões de consumo de álcool na população brasileira. Laranjeira R... [et al]. Brasília: Secretária Nacional Antidrogas; 2007. p.75.

6. Brasil. Ministério da Educação. Ministério da Saúde. Gabinete de Segurança Institucional da Presidência da República. Ministério da Justiça. Ministério do Desenvolvimento Social. Decreto 7.170/2010. Brasília 2010; 6p.

7. Brasil. Ministério da Justiça. Ministério da Fazenda. Ministério da Educação. Ministério da Educação. Ministério do Desenvolvimento Social e Combate à Fome. Casa Civil da Presidência da República. Decreto 7.637/2011. Brasília 2011; 8p.

8. Bucher R. Drogas e drogadição no Brasil. Porto Alegre: Artes Médicas; 1992.

9. Freud S. O mal estar na civilização. Edição Standard Brasileira das Obras Psicológicas Completas. V 21. Rio de Janeiro: Imago; 1976.

10. Sabino NM, Cazenave SOS. Comunidades terapêuticas como forma de tratamento para a dependência de substâncias psicoativas. Arq est psi. 2005; 22(2): 167-174.

11. Silveira CM. Padrões de Consumo do Álcool na População Brasileira. In: Brasil. Ministério da Justiça. Secretaria Nacional de Políticas sobre Drogas (SENAD). Prevenção do Uso de Drogas. $5^{\underline{a}}$ ed. Brasília, DF: SENAD; 2013. p. 89.

12. Schenker M. Drogas, Individuo, Família e Sociedade Paradigma Sistêmico. In Inem, Baptista C , Marcos (orgs.). Toxicomanias: abordagem clínica. 1를 ed. Rio de Janeiro; 1997.

13. DSM-IV-TR. Manual diagnóstico e estatístico de transtornos mentais. Trad. Cláudia Dornelles. 4를 ed. Porto Alegre: Artmed; 2002.

14. Almeida ACA, Pereira AS, Reis AV, Tabosa MS, Andrade KR, Almeida LM, Viana MP, et al. Proteção Social Básica: a importância da dimensão preventiva do uso e dependência do crack e outras drogas. 2005; 26(3): 21-42.

15. Brasil. Sistema Único de Saúde [homepage na Internet]. Legislações; 2013.

16. Conselho Federal de Psicologia. [homepage na Internet]. Avaliação das Comunidades Terapêuticas.

17. Brasil. Ministério da Saúde. Agencia Nacional de Vigilância Sanitária. Diretoria Colegiada da Agência Nacional em Vigilância Sanitária. Resolução-Rdc/Anvisa no 101/2001. Brasília; $2001 ; 15 p$. 
18. Cruz MS. Redução de Danos, Prevenção e Assistência. In: Brasil. Ministério da Justiça. Secretaria Nacional de Políticas sobre Drogas (SENAD). Prevenção do Uso de Drogas. 5르. ed. Brasília, DF; 2013. p. 163-4.

19. Secretaria Nacional Antidrogas. [homepage na internet]. Comunidades Terapêuticas. 2013.

20. Bós ÂJG. Epi Info sem mistérios: um manual prático. Porto Alegre: EDIPUCRS, 2012. p. 211.

21. Brasil. Ministério da Saúde: Agência Nacional de Vigilância Sanitária. RESOLUÇÃO - RDC № 29, DE 30 DE JUNHO DE 2011.

22. Perrone P A K. A comunidade terapêutica para recuperação da dependência do álcool e outras drogas no Brasil: mão ou contramão da reforma psiquiátrica?. Ciênc. saúde coletiva [online]; 2014, (19):2, p. 569-

23. Costa SF. As políticas públicas e as comunidades terapêuticas nos atendimentos à dependência química. Serviço Social em Revista. Departamento de Serviço Social da Universidade Estadual de Londrina. Jan/Jun; 2009, (11): 2. p.01-14.

24. Scaduto AA. O tratamento de dependentes de substâncias psicoativas numa comunidade terapêutica: estudo através da avaliação psicológica. Dissertação (Mestrado) - Universidade de São Paulo, Ribeirão Preto; 2010, $170 f$.

25. Schnorr A, Hess ARIB. Perspectivas do usuário de crack ao término do tratamento em comunidades terapêuticas quanto a sua reinserção social. Trabalho de Conclusão II. Artigo de pesquisa. Curso de Psicologia das Faculdades Integradas de Taquara,Taquara-RS; 2012.

26. Pantoja DJS. As dificuldades enfrentadas pela estratégia saúde da família no cuidado à saúde do dependente químico. Daumas H. Dissertação (Mestrado em Saúde da Família). Universidade Estácio de Sá, 2012. Rio de Janeiro, 2012. $78 f$.

27. Barros CAS. Um estudo sobre relações entre indivíduo, comunidade e desenvolvimento local a partir de uma comunidade terapêutica, Tese (Mestrado em Desenvolvimento Local) Universidade Católica Dom Bosco. UCDB de Campo Grande. Programa de Pós-Graduação em Desenvolvimento Local. Campo Grande; 2012. 68 .

28. Costa FS. O processo de reinserção social do dependente químico após completar o ciclo de tratamento em uma comunidade terapêutica. [Internet]. 2000 [acesso em 2014 nov 4]; 3(2): 30 p. Disponível em: http://www.uel.br/revistas/ssrevista/c_v3n2_processo.htm. 